\title{
Measurements of Transition Probabilities for Complex Ions
}

\author{
H. Nilsson ${ }^{1}$, S. Ivarsson ${ }^{1}$, H. Sabel ${ }^{1}$, C. M. Sikström ${ }^{1}$ and L. J. Curtis ${ }^{2}$ \\ ${ }^{1}$ Atomic Astrophysics, Lund Observatory, Lund University, Box 43, SE-221 00 Lund, Sweden \\ ${ }^{2}$ Department of Physics and Astronomy, University of Toledo, Ohio 43606, USA \\ Received October 1, 2002; accepted October 29, 2002 \\ PACS Ref: $32.30 .-\mathrm{r}, 30.32 . \mathrm{Cs}$
}

\begin{abstract}
Fourier transform spectroscopy has proven to be a useful tool when measuring wavelengths and branching fractions. The high spectral resolution makes it possible to extend and improve term analyses of complex spectra. We present an overview of our measurements with the Fourier transform spectrometer at Lund Observatory. Much of our work is motivated by astrophysical problems, and we show an application of some of our measurements related to the age determination of the Galaxy.
\end{abstract}

\section{Introduction}

Most of our knowledge about the Universe has emerged from observations of the light radiated by various cosmic objects. In order to analyze this light atomic parameters such as wavelength, energy level, oscillator strength and line broadening effects have to be known from laboratory measurements.

The new generation of high-resolution spectrographs used in modern astrophysics defines the present demands on atomic data. Space instruments like the Goddard High Resolution Spectrograph (GHRS) and its successor, the Space Telescope Imaging Spectrograph (STIS), on board the Hubble Space Telescope (HST) have greatly increased the demands for atomic data in the far ultraviolet region. Its predecessors, the Copernicus and International Ultraviolet Explorer (IUE) satellites clearly demonstrated the needs for new ultraviolet atomic data, but analyses of HST spectra demanded higher accuracy of nearly all atomic data. Recent, present and future space observatories in the infrared, like the Atmospheric Trace Molecule Spectroscopy Experiment (ATMOS), the Infrared Space Observatory (ISO) and the Stratospheric Observatory for Infrared Astronomy (SOFIA), and in the X-ray regions (CHANDRA) have expanded the request for atomic data in wavelength regions previously unexplored. Finally, with the use of the Ultraviolet Echelle Spectrograph (UVES) on the Very Large Telescope (VLT) the time has come for a revision of the atomic data in the optical region, which can be observed from ground.

In an astrophysical spectrum wavelengths are necessary for line identification and the energy levels for the corresponding electronic transition. The transition probabilities are used to derive the cosmic abundances of the different elements by using stellar spectra.

Laboratory wavelengths can be measured with a small uncertainty $\left(<1\right.$ part per $\left.10^{7}\right)$, and energy levels can be determined from the wavelengths with a corresponding uncertainty. Transition probabilities for complex spectra on the other hand are difficult to measure better than $10 \%$.

\section{Experimental equipment}

The laboratory work in the Atomic Astrophysics group at Lund Observatory is based on a Chelsea Instrument FT500 Fourier transform (FT) spectrometer (experimentally) designed for the ultraviolet wavelength region.

The FT spectrometer is based on the principle of the Michelson interferometer. Among its advantages are a high signal-to-noise ratio (SNR) and a large dynamic intensity range making it possible to measure a large span of line intensities. All sample points include information of all wavenumbers. The high spectral resolution provides accurate wavenumbers and decreases the risk of erroneous identifications and blends with other lines. It is also possible to record large spectral intervals on a linear wavenumber scale. One of the major disadvantages is that one can only record spectra from unpulsed and stable light sources, which limits the studies of an element to its three lowest ionization stages, i.e., to recordings of mainly first, second and in some cases third spectra.

As light sources we use a hollow cathode (HC) lamp and a Penning discharge (PD) lamp.

\section{Energy levels}

The basic feature in the physics of atoms is the energy level structure, which is derived by an analysis of the observed spectrum, the term analysis. There is little of diagnostic value in a spectral line whose identity is not known. The term analysis is not only guided by theoretical predictions but also by other tools. For example, differences in the excitation of the spectrum in different light sources and regularities along isoelectronic and isoionic sequences may provide important information for the analysis. There are some specific aspects of the knowledge about atomic structure for pure astrophysical applications. One needs more information about the spectra and structure of abundant elements than of trace elements. One needs more information about the highly excited states when doing an analysis of a stellar spectrum in the satellite ultraviolet region, simply because the upper level of an ultraviolet absorption line has higher excitation energy than the upper level of an optical line. This has been the main problem when analysing spectra from the HST spectrographs [1].

The first step in the term analysis is to record the spectrum and make a wavelength calibrated linelist. In order to reduce the number of lines in the linelist, "impurity" lines are removed. Such lines may originate from the carrier gas and from other ionization stages than 
the one under investigation. Spectra recorded under various discharge conditions of the light source or even with different light sources can sometimes be used to distinguish between different ionization stages and even levels of excitation.

The previously known spectral lines in the spectrum under investigation are used to derive improved energy values for the known levels. The classified lines are removed from the linelist and placed in a separate list. The search for new energy levels is performed with a computer program (LEVELS), requires known energy levels and the wavenumbers for unidentified lines as input. It adds the wavenumber of each unidentified line $\left(\sigma_{i}\right)$ to a level $E_{1}$, repeats the same procedure for level $E_{2}$ and continues for all known levels inserted in the input list. The resulting sums $\left(E_{k} \pm \sigma_{i}\right)$ are sorted according to wavenumber, and the code saves each group of sums, for which the maximum difference between adjacent sums is less than $\delta E$, i.e.:

$\delta E>\left(\sigma_{1}+E_{1}\right)-\left(\sigma_{2}+E_{2}\right)$.

Such a group of sums might be a potential energy level, and an example of the output from the program showing a real energy level is shown in Table I. The entries are the Energy, the Wavenumber and Intensity of the observed lines in the list of unidentified lines, name (Level) and $J$ value of the combining levels. The right hand column (New Level) gives the sums defined above.

The values of $\delta E$ and the least number of coincidences in a group of sums can be set. Groups identified as new levels are added to the list of energy levels and the corresponding identified lines are moved to the list of identified lines. This procedure can now be repeated with the new energy levels included in the energy level list used as input to LEVELS. The final energy values are calculated with a computer code ELCALC [2] that optimizes the energy level values from the experimental wavenumbers of the spectral lines.

To get an idea of where to look for new levels and what transitions to expect, theoretical predictions can be consulted. Such predictions can be made by means of calculations with the Cowan code [3]. The advantage of the Cowan code compared with other more sophisticated codes is that it is reasonably easy to use and easily gives $f$-values for a large number of lines.

The labeling of new energy levels can usually be solely based on the observed transitions, but if the level is severely mixed it may be difficult to assign a proper label to the level. In such cases it is useful to consult theoretical calculations and assign the largest eigenvector to the mixed level. In complex spectra of heavy elements where $L S$ coupling fails to give a proper description of the structure the $L S$ name of a level has little physical meaning. However, it is often practical to assign a name to each new level.

\section{Wavelengths}

The basis for the term analysis of a spectrum is the measurement of wavelengths, as the wavenumbers of the spectral lines are used to build up the energy level system. The search for new energy levels by using the line list is seldom unambiguous, and the degree of ambiguity depends on the accuracy of the wavelengths. Even though many extensive term analyses are based on spectra recorded at low dispersion, the analyses contain few doubtful identifications. However, extensions of these analyses require in general spectra at high resolution and high dispersion, which can be achieved with the FT spectrometer. Thus, for extensions and improvements of the energy level diagrams we have used new wavelength measurements from the Lund FT spectrometer. As mentioned above this instrument has its limitations in wavelength coverage and discharge conditions of the light source.

The quality of the astrophysical spectra recorded with modern ground-based and space-borne telescopes, like the VLT and the HST, has shown that the accuracy of laboratory wavelengths is unsatisfactory for many spectra. Besides the internal needs for accurate wavelengths in the term analysis, there is thus also a need for higher accuracy in the applications to astrophysical spectra. There are numerous examples found in the " $\chi$ Lupi Pathfinder Project" [4,5] and [6], where the HST spectrum of a sharp-lined star reveals inaccurate laboratory wavelengths.

A FT spectrum has a linear wavenumber scale which is defined by the $\mathrm{He}-\mathrm{Ne}$ laser line. However, to obtain a higher level of accuracy one has to correct for a linear wavenumber shift, which arises due to a slight difference in optical path length between the sampling laser and the light from the light source. The wavenumber uncertainty in FT spectra are usually between 0.003 and $0.030 \mathrm{~cm}^{-1}$ depending of the SNR and shape of the line.

Table I. Output from the LEVELS program, showing the transitions to $\left({ }^{5} \mathrm{D}\right) 5 d \mathrm{e}^{6} \mathrm{~F}_{9 / 2}$ in Mo II.

\begin{tabular}{llccccc}
\hline Energy & & Wavenumber & Intensity & Level & $J$ & New Level \\
\hline 52217.587 & + & 35343.355 & 225 & 5D)5p z4F3 & 3.5 & 87560.942 \\
52843.296 & + & 34717.649 & 733 & 5D)5p z4F4 & 4.5 & 87560.945 \\
50302.913 & + & 37258.032 & 3587 & 5D)5p z6D3 & 3.5 & 87560.945 \\
49481.300 & + & 38079.646 & 7421 & $5 \mathrm{D}) 5 \mathrm{p}$ z6P3 & 3.5 & 87560.946 \\
47999.856 & + & 39561.090 & 7644 & 5D)5p z6F4 & 4.5 & 87560.946 \\
48960.043 & + & 38600.903 & 4415 & $5 \mathrm{D}) 5 \mathrm{p}$ z6F5 & 5.5 & 87560.946 \\
50705.942 & + & 36855.005 & 15869 & 5D)5p z6D4 & 4.5 & 87560.947 \\
52843.296 & + & 34717.652 & 699 & 5D)5p z4F4 & 4.5 & 87560.948
\end{tabular}




\section{Transition probabilities}

The intensity of a spectral line given in various linelists often represents different quantities and are in most cases given on a relative scale. For emission lines it could represent the integrated flux of the line profile, even if it in many cases stands for the peak intensity. In stellar absorption line spectra the equivalent width is mostly used. In any case, the intensity is determined by the number of atoms/ions in the initial atomic level of the transition and the transition probability $(A)$ or oscillator strength $(f)$. There is a simple relation between these two quantities, but most astrophysical formulae and codes use oscillator strength, or the $\log g f$-value, where $g$ is the statistical weight of the initial energy level. Thus, if one wants to use the stellar spectrum to derive the relative abundance of an element in the star's atmosphere the oscillator strengths of the lines have to be known.

There are several different ways to measure oscillator strengths in the laboratory, directly by e.g., stabilized are measurements (emission) or applying the hook method (absorption), and indirectly by combining measurements of branching fractions (emission) and radiative lifetimes. Emission and absorption measurements can be combined in the so called "leap frogs" and "bow ties". A comprehensive review of the different methods is given by [7]. Relative $f$-values of lines at wavelengths shortwards of $2000 \AA$ have been measured in absorption using synchrotron radiation as a background source [8].

In our measurements of $f$-values we combine radiative lifetimes, measured at Lund Laser Centre, and branching fractions (BFs) measured with our FT spectrometer. The lifetime is measured with the laser induced fluorescence (LIF) method, where the state is selectively photoexcited in one, two or three steps by tunable lasers. Two steps are used if the state under investigation has the same parity as the state, from which the excitation starts, i.e., normally the ground state. Three steps are used if the state cannot be reached in one step from the initial state of the excitation scheme.

\subsection{Branching fractions}

The BF of a line is defined as the transition probability, $A_{u l}$, of a single line divided by the sum of all transition probabilities from the same upper level $E_{u}$. The inverse of this sum is equivalent to the lifetime, $\tau_{u}$, of the upper level. The measured quantity is the intensity $\left(I_{u l}\right)$, which is proportional to the population $\left(N_{u}\right)$ and the statistical weight $\left(g_{u}\right)$ of the upper level and $A_{u l}$, i.e.,

$I_{u l} \propto N_{u} g_{u} A_{u l}$.

The BF can be expressed as

$(B F)_{u l}=\frac{A_{u l}}{\sum_{l} A_{u l}}=\frac{I_{u l}}{\sum_{l} I_{u l}}$

where $I_{u l}$ is the measured intensity corrected for the instrument response. Since

$\tau_{u}=\frac{1}{\sum_{l} A_{u l}}$ one gets

$A_{u l}=\frac{(B F)_{u l}}{\tau_{u}}$,

which shows the relation between the transition probability, branching fraction and radiative lifetime $\tau_{u}$. The transition probability can be converted to an oscillator strength through (see e.g., [9])

$f=\frac{\varepsilon_{0} m c^{3}}{2 \pi e^{2} v^{2}} \frac{g_{u}}{g_{l}} A_{u l}$

where $g_{u}$ and $g_{l}$ are the statistical weights of the upper and lower levels, respectively. Inserting the constants gives the relation

$f=1.499 \cdot 10^{-16} \frac{g_{u}}{g_{l}} \lambda^{2} A_{u l}$,

where $\lambda$ is in $\AA$ and $A$ is in $\mathrm{s}^{-1}$.

The uncorrected intensities of the lines are measured by fitting Voigt profiles to the recorded line profiles. In order to get relative intensities for the individual branches at different wavelengths the recorded spectra have to be corrected for the instrument response (see Fig. 1). Two different continuum lamps are used for this purpose in the measurements made in Lund: A deuterium lamp calibrated between 1600 and $4100 \AA$ and a tungsten ribbon lamp calibrated between 3000 and $7000 \AA$. However, the disadvantage of using continuum lamps is that they provide an external calibration. They are mounted separately and if the alignment differs from that of the light source under investigation, the errors introduced can be significant. This problem can be avoided by an internal calibration, which is provided by experimental branching ratios (BR) for carrier gas lines present in the same spectrum as the lines being studied. There exist numerous sets of argon lines having common upper levels which are well distributed in wavelength and thus suitable as internal calibration lines [10]. A combination of the continuous radiation curve from a continuum lamp and the internally known branching ratios produces a satisfactory response curve for the intensity calibrations.

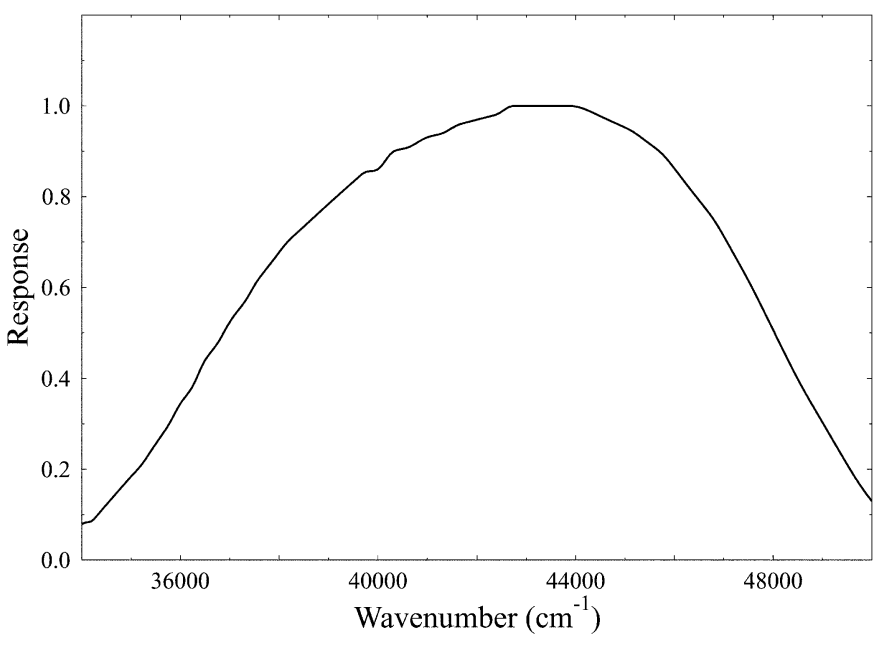

Fig. 1. An example of the instrument response of the FT spectrometer derived from a deuterium calibration lamp. 
A serious problem using emission line intensities for the $\mathrm{BFs}$ arises when one or more branches combines with the ground state or very low states, as they may be affected by self absorption. In order to get reliable BFs and small uncertainties in such cases it is crucial to check and correct for self absorption. This can be done by measuring the intensity ratio of two lines from the same upper level at different plasma conditions, which are changed by varying the current through the light source. By plotting the intensity ratio against the discharge current one can study effects of self absorption and corrections can be made. If the ratio is independent of the plasma density, the lines are not self absorbed, whereas one or both lines are affected by self absorption if the ratio changes with the current. The ratio of the lines is then extrapolated to zero current, where it is assumed to be free from self absorption (see [11]).

For most complex spectra the intensity of some lines from an upper level can not be measured, either because they are too weak or they fall outside the wavelength interval covered. The total intensity or branching fraction of these missing lines is called the residual, which can often be satisfactorily estimated from theoretical calculations. The quality of the theoretical calculations can be estimated from a comparison of the predicted and measured BR values of the observed lines. Also, the relevant quantity is the size of the residual relative to the individual BFs of the significant lines. When possible, it is thus meaningful to estimate and report the amount of the residual intensity for each energy level.

In many cases not all lines from an upper energy level under study fall in a wavelength interval narrow enough to be recorded in one FT spectrum. Different spectra have to be combined in order to measure all lines, and overlapping lines are used to transfer the calibration between the different spectra. In principle, it should be possible to arbitrarily choose the overlapping lines, but, since the plasma conditions may change, it is recommended that lines from the measured level are used. If this is not possible great care should be taken to assure that the plasma conditions are unchanged between the different recordings. A typical uncertainty introduced by combining spectra is between 5 and $10 \%$.

In [11] we discuss how to derive the uncertainty of the value of an oscillator strength, obtained by combining measured lifetimes and branching fractions. The individual uncertainties contributing to the final uncertainty are combined with a propagation of error formula, which treats all contributing uncertainties as uncoupled. The individual uncertainties included in this formula are due to: the intensity measurement, the calibration of the continuous lamps, the intensity calibration, the combination of different spectra, the lifetime measurements, the self absorption correction, and the estimate of the residual.

\subsection{Lifetime measurements}

Radiative lifetimes have been measured using the LIF method, see e.g., [12], at the Lund Laser Centre. In order to populate the energy level under investigation a laser produced plasma is created by directing laser pulses onto a solid target of the element under studie. In order to selectively pump the ions in the plasma a tunable dye laser is used. When measuring short lifetimes the excitation pulses can be shortened by applying stimulated Brillouin scattering [13]. In this way the pulses can be compressed from 10 to $1 \mathrm{~ns}$. In some measurements the Stoke's shift in a $\mathrm{H}_{2}$ cell is utilized to shift the pulse to the desired wavelength. The fluorescence is selected with a $0.12 \mathrm{~m}$ monochromator and detected with a photo-multiplier tube, and recorded with a transient recorder. A more detailed description of the laser system can be found in [12].

\section{The FERRUM Project}

A general problem with astrophysical analyses of stellar spectra in the satellite ultraviolet region has been the lack of experimental oscillator strengths for abundance work. This has been clearly demonstrated by the Hubble Space Telescope in the $\chi$ Lupi Pathfinder Project ([6] and references therein). For example, the first experimental oscillator strengths for Fe II lines below $2000 \AA$ were published in 1996 [14]. In the resonance region 2000$3000 \AA$ only a few transitions to the lowest excited states have experimental $g f$-values measured by combining BFs and radiative lifetimes. The relatively high cosmic abundance of iron and the complex atomic structure of Fe II make ultraviolet stellar spectra crowded with Fe II lines. In an effort to extend the database of reliable oscillator strengths, an international ongoing collaboration, The FERRUM Project [15], has been initiated in Lund. The FERRUM Project aims to measure oscillator strengths for transitions both in the ultraviolet and optical wavelength regions and with a spread in excitation potential of the lower energy level.

The half-filled d-shell and the fact that three configurations of the same parity are competing in occupying the ground state, make the atomic structure of Fe II complex. The ground configuration is $3 \mathrm{~d}^{6} 4 \mathrm{~s}$, and the competing configurations are $3 \mathrm{~d}^{7}$ and $3 \mathrm{~d}^{5} 4 \mathrm{~s}^{2}$. The fundamental transitions (with thousands of strong lines) appear in the satellite UV region: $4 \mathrm{~s}-4 \mathrm{p}$ at $2500 \pm 200 \AA$ and $3 \mathrm{~d}-4 \mathrm{p}$ starting at $1600 \AA$ for the normal system, $3 \mathrm{~d}^{6}\left({ }^{M} L\right) n l$, and all wavelengths shifted shortwards for the doubly-excited system, $3 \mathrm{~d}^{5}\left({ }^{M} L\right) n \ln ^{\prime} l^{\prime}$. As can be seen in Fig. 2, the transition energies for the $4 \mathrm{p}-5 \mathrm{~s}$ and $4 \mathrm{p}-4 \mathrm{~d}$ arrays in the normal system are nearly the same as for $4 \mathrm{~s}-4 \mathrm{p}$, making the Fe II spectrum extremely line rich between 2000 and $3000 \AA$. The simplified diagram of a small part of the total energy level structure in Fe II shown in Fig. 2 illustrates the present status of The FERRUM Project. The $n l$ boxes represent groups of energy levels, which belong to the lowest subconfigurations of the normal system, $3 \mathrm{~d}^{6}\left({ }^{5} \mathrm{D}\right) \mathrm{nl}$, i.e., they are built on the ${ }^{5} \mathrm{D}$ parent term of the $3 \mathrm{~d}^{6}$ configuration in Fe III. The $n l^{\prime}$ boxes represent energy levels belonging to other subconfigurations of the normal system, i.e., the parent term ${ }^{M} L \neq{ }^{5} \mathrm{D}$. The $4 \mathrm{~s}^{2}$ and $4 \mathrm{~s} 4 \mathrm{p}$ boxes represent parts of the $3 d^{5} 4 s^{2}$ and $3 d^{5} 4 s 4 p$ configurations, respectively, which belong to the doubly-excited system.

In The FERRUM Project we have so far measured oscillator strengths for a number of transitions from each array indicated with arrows in Fig. 2. Previously there have mainly been measurements of oscillator strengths for lines originating from the lowest excited subconfiguration of odd parity, $3 d^{6}\left({ }^{5} D\right) 4 p$, i.e., $4 s-4 p$ and $4 s^{\prime}-4 p$ transitions 


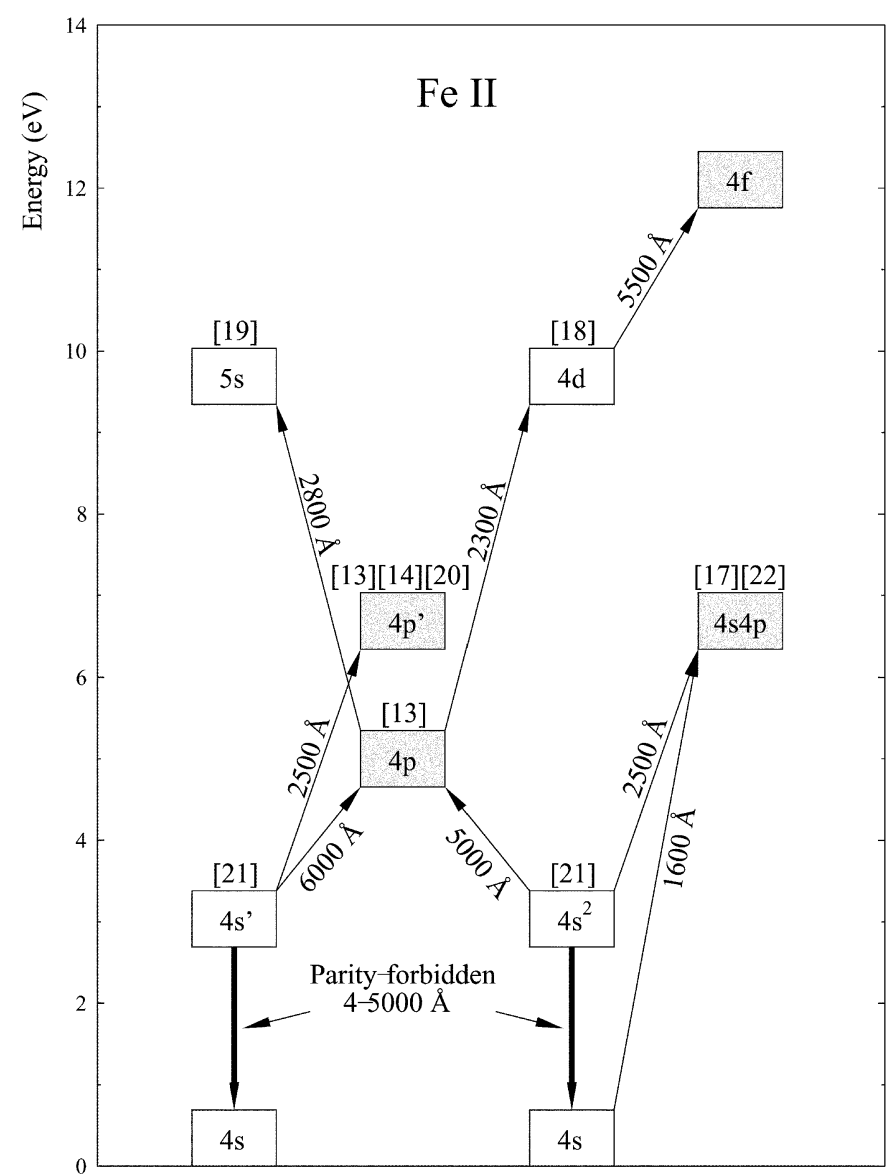

Fig. 2. Schematic diagram over a part of the level structure in Fe II showing the status of The FERRUM Project. White boxes indicate even configurations and gray boxes odd configurations. The references to the papers including $\mathrm{BF}$ and lifetime measurements for levels within the configuration are given above the boxes.

using the notation from Fig. 2. Only a few oscillator strengths for lines between $4 s^{\prime}$ and $4 p^{\prime}$ have been measured with the emission technique. Most of the $4 p$ levels have transitions both to the ground term and to low metastable levels. The ground term transitions are usually not suitable for abundance analysis since they are often saturated in stellar spectra, and there is thus a need for data for weak lines. Weak stellar lines are more suitable for abundance analyses but weak transitions are difficult to measure reliably in the laboratory if their upper level also has transitions down to the ground term. When the iron density in the laboratory source is high enough for the weak lines to show up, the strong lines are optically thick. On the other hand, when the strong lines are optically thin, the weak lines vanish or become too weak, and the uncertainties in the measurements increase drastically. However, a strong line with high $g f$-value can be produced as a weak line in the stellar spectrum, if the lower level has a low population, i.e., a sufficiently high excitation potential. The study of such lines is one of the goals of The FERRUM Project.

The nine papers in The FERRUM Project published so far are: lifetimes of four levels, two $4 p$ and two $4 p^{\prime}$ levels [12]; lifetimes of six $3 d^{6} 5 p$ levels, and 18 branching fractions from two of the levels [16]; lifetimes of the three $4 s 4 p y^{6} \mathrm{P}$ levels [17]; the lifetime of six $3 d^{6} 4 d$ levels are reported together with 29 branching fractions [18]; oscillator strengths for $204 \mathrm{p}-5 \mathrm{~s}$ transitions from four $5 \mathrm{~s}$ levels [19]; $g f$-values for 81 lines from four of the levels reported in [16,20]; lifetimes of two metastable levels $\left(4 s^{\prime}\right.$ and $4 \mathrm{~s}^{2}$ ) are reported in [21]; a review paper with a status report of The FERRUM Project [15]; 19 branching fractions from the three levels reported in [17,22].

So far, 167 transition probabilities and 25 lifetimes have been reported for Fe II within The FERRUM Project, and work is in progress concerning lifetimes and absolute transition probabilities for $4 \mathrm{~d}-4 \mathrm{f}$ transitions in Fe II.

The next step is to extend The FERRUM Project to other iron-group elements, and measurements have started on titanium and nickel.

\section{Cosmochronology}

Two complex ions for which experimental $f$-values have become important for an astrophysical application are U II and Th II, although the abundance of both thorium and uranium is low in stars. The reason for the astrophysical interest is that they are radioactive and can serve as cosmological clocks. Thorium and uranium are rapid neutron capture (r-process) elements and therefore believed to be produced in environments with high neutron flux, e.g., in supernova $(\mathrm{SN})$ explosions.

The idea behind cosmological dating is to compare the present abundance ratio of two elements (where at least one is radioactive) with the ratio at the time when the star was formed. The present value is determined through spectral analysis of the star, while the value at the star formation epoch has to be estimated with theoretical predictions of the production ratios. To check the quality of the calculations one can for example compare the predictions of stable r-process elements with the measured abundances in the sun. Even if the predicted abundance ratio for stable isotopes is good the uncertainties for the radioactive elements can be large. To minimize the uncertainties in the calculation of the production ratio one should use isotopes that have about the same mass and therefore have about the same systematic errors in the calculations.

Uranium has been found in some CP stars (e.g., [23] and [24]), but because of the unknown mechanisms behind the enhancement of some heavy elements, these stars are not good candidates for cosmochronology [25]. Thorium has on the other hand been used for cosmochronology. In e.g., [26] the abundance ratio of thorium and europium was used to determine the age of a sample of metal-poor halo stars. A problem with thorium is that the half-life is of the same order as the age of these stars (and the age of the Universe) and less sensitive in the dating than uranium. In 2001 the detection of a U II line was reported in the spectrum of the star CS31082-001 [27]. The paper also included an age determination of the star based on a cosmochronometer using thorium and uranium. Since uranium has a half-life of $4.5 \mathrm{Gyr}$ this gives a more sensitive measure than the $\mathrm{Th}-\mathrm{Eu}$ cosmochronometer based on the thorium half-life of 14.1 Gyr. CS31082-001 is a metal poor halo star with a low iron abundance (about $1 / 1000$ compared to the sun), indicating that the star is old and it has an enhancement of r-process elements. The age of CS31082-001 can therefore be used to put a lower limit on the age of the Galaxy. 
The age of CS31082-001 determined with the uraniumthorium cosmochronometer reported in [27] was $12.5 \pm 3.0$ Gyr, but there seemed to be a problem with the oscillator strengths used in the abundance determinations. For the $\mathrm{U}$ II line the experimental values given in the literature differed by about a factor of three, from $g f=0.19$ to 0.63 $[28,29,30,31,32,33]$ and [34]. The oscillator strengths in Th II date back to the measurements reported in [28], rescaled in different ways in [30] and [35]. New measurements of oscillator strengths in both U II and Th II were thus needed in order to decrease the uncertainties in the age determination of CS31082-001.

The age of the star CS31082-001 has been redetermined using the new laboratory data reported in $[36,37]$ and [38], and the revised value is $14.0 \pm 2.4$ Gyr [25].

\section{Summary}

Atomic data for complex atoms and ions are of great interest to the astronomy community in order to study the evolution of the Universe. Fourier transform spectroscopy is one way to produce these kind of data.

\section{Acknowledgements}

HN would like to thank Prof. I. Martinson for making the trip to Sofia possible. The authors would like to thank Prof. S. Johansson for his help with the manuscript.

\section{References}

1. Johansson, S., Leckrone, D. S. and Davidson, K., Astron. Soc. Pac. Conference Series 143, 155 (1998).

2. Radziemski, L. J., Fischer, K. L., Steinhaus, D. W. and Goldman, A. S., Comp. Phys. Comm. 3, 9 (1973).

3. Cowan, R. D., "The Theory of Atomic Structure and Spectra," (University of California Press, Berkeley, 1981).

4. Johansson, S. G., Joueizadeh, A., Litzén, U. et al., Astrophys. J. 421, 809 (1994).

5. Johansson, S., Kalus, G., Brage, T., Leckrone, D. S. and Wahlgren, G. M., Astrophys. J. 462, 943 (1996).

6. Leckrone, D. S., Proffitt, C. R., Wahlgren, G. M., Johansson, S. G. and Brage, T., Astron. J. 117, 1454 (1999).

7. Huber, C. E. and Sandeman, R. J., Rep. Prog. Phys. 49, 397 (1986).
8. Mullman, K. L., Sakai, M. and Lawler, J. E., Astron. Astrophys. 122, 157 (1997)

9. Thorne, A. P., Litzén, U. and Johansson, S., "Spectrophysics, Principles and Applications," (Springer-Verlag Berlin, Heidelberg, 1999).

10. Whaling, W., Carle, M. L. and Pitt, P.L., J. Quant. Spectrosc. Radiat. Transfer 50, 7 (1993).

11. Sikström, C. M., Nilsson, H., Litzén, U., Blom, A. and Lundberg, H., J. Quant. Spectrosc. Radiat. Trans. 74, 355 (2002).

12. Li, Z. S., Lundberg, H., Sikström, C. M. and Johansson, S., Eur. Phys. J. D 6, 9 (1999).

13. Li, Z. S. et al., Phys. Rev. A 60, 198 (1999).

14. Bergeson, S. D., Mullman and Lawler, J. E., Astrophys. J. 464, 1044 (1996).

15. Johansson, S. et al., Physica Scripta T100, 71 (2002).

16. Sikström, C. M. et al., J. Phys. B: At. Mol. Opt. Phys. 32, 5687 (1999).

17. Li, Z. S, Lundberg, H., Berzinsh, U., Johansson, S. and Svanberg, S., J. Phys. B: At. Mol. Phys. 33, 5593 (2000).

18. Nilsson, H. et al., Astron. Astrophys. 362, 410 (2000).

19. Karlsson, H., Sikström, C. M., Johansson, S., Li, Z. S. and Lundberg, H., Astron. Astrophys. 371, 360 (2001).

20. Pickering, J. C., Johansson, S. and Smith, P. L., Astron. Astrophys. 377, 361 (2001).

21. Rostohar, D. et al., Phys. Rev. Lett. 86, 1466 (2001).

22. Pickering, J. C., Donnelly, M., Nilsson, H., Hibbert, A. and Johansson, S., Astron. Astrophys. (in press).

23. Jaschek, M. and Malaroda, S., Nature 255, 246 (1970).

24. Cowley, R. C., Aikman, G. C. C. and Fisher, W. A., Publ. Dom. Astrophys. Obs. Victoria 15, 37 (1977).

25. Hill, V. et al., Astron. Astrophys. 387, 560 (2002).

26. Cowan, J. J. et al., Astrophys. J. 521, 194 (1999).

27. Cayrel, R. et al., Nature 409, 691 (2001).

28. Corliss, C. H. and Bozman, W. R., NBS Monograph, Vol. 53 (Washington DC: US Govt. Printing Office, 1962).

29. Voigt, P. A., Phys. Rev. A11, 1845 (1975).

30. Corliss, C. H., J. Res. Nat. Bur. Std. 80A, 429 (1976).

31. Palmer, B. A., Keller, R. A. and Engleman, Jr., R., LA-8251-MS Los Alamos Internal Report (1980).

32. Chen, H.-L. and Borzileri, C., J. Chem. Phys. 74, 6063 (1981).

33. Compant La Fontaine, A., J. Phys. B: At. Mol. Phys. 20, 6649 (1987).

34. Henrion, G., Fabry, M. and Remy, M., J. Quant. Spectrosc. Radiat. Transfer 37, 477 (1987).

35. Simonsen, H., Worm, T., Jessen, P. and Poulsen, O., Physica Scripta 38, 370 (1988).

36. Lundberg, H., Johansson, S., Nilsson, H. and Zhang, Z., Astron. Astrophys. 372, L50 (2001).

37. Nilsson, H., Ivarsson, S., Lundberg, H. and Johansson, S., Astron. Astrophys. 381, 1090 (2002).

38. Nilsson, H., Zhang, Z. G., Lundberg, H., Johansson, S. and Nordström, B., Astron. Astrophys. 382, 368 (2002). 